\title{
Perceptual organization of nine-element auditory temporal patterns
}

All possible basic patterns formed from repeating sequences of nine dichotomous elements were presented auditorily, and Ss were required to describe the sequence after it became heard as an organized pattern. These descriptions show that whenever a particular pattern is a preferred organization, so also is its temporal reversal, whether that temporal reversal occurs in the same or in another basic pattern. Thus, it is argued that the pattern organizations are wholistic. The major organizational priniciples are: preference for (a) patterns that are balanced in time with long runs at the ends, and $(b)$ patterns that have a directional simplicity with nun lengths either increasing or decreasing in a regular order. Variability of pattern organization is related to the number of runs in the sequence, the range of run lengths represented, and the availability of a preferred organizing principle. It is concluded that the onganism selects a pattern or organization from a set of alternatives presented by the structural features mentioned, and that this organizational selection is made on the basis of the whole pattern, not on parts of it built up sequentially.

In recent years, there has developed considerable interest in the learning and perception of repeated fixed sequences of elements, usually, but not always, dichotomous. Interest in the problem has exhibited three different facets, facets that can be studied independently, although hopefully all will ultimately converge to a common understanding of the psychological nature of temporal pattern perception and learning.

The most common approach has been to study the difficulty with which different temporal patterns are learned, with the intent of understanding the stimulus properties that lead to difficulty, and therefore of understanding the psychological processes involved in the learning. When stimuli are presented at slow rates, with seconds between successive events, learning difficulty is measured (e.g., Restle, 1967; Vitz, 1968; Vitz \& Todd, 1967; Galanter \& Smith, 1958; Garner \& Gottwald, 1967). Royer and Garner (1966), using stimulus-presentation rates too fast for learning of individual elements to occur but not too fast for apprehension as a pattern which then could be reproduced motorically, used a delay of perceptual organization as a measure of pattern difficulty; and Garner and Gottwald (1968) showed that, even though the learning of slow patterns involves some different psychological processes from the perception of faster patterns, similar principles of perceptual organization operate for both types of performance. Thus, patterns that are difficult to learn at slow rates are also difficult to perceive in an organized fashion at higher rates.

A second approach to the problem has been the direct study of the perceived organization of sequences of dichotomous elements. When sequences of dichotomous elements are repeated indefinitely without interruption, there are as many possible beginnings to the perceived pattern as there are elements in it. Thus, XXO, when repeated (XXOXXOXXO), is not distinguishably different from $\mathrm{XOX}$ or OXX except for the starting point. Yet, when such a sequence is learned or perceived, any of the three possible organizations is the one that could be learned, and this second approach aims at discovering these preferred organizations. Royer and Garner (1966), using buzzers as dichotomous elements to generate sequences of eight elements, found that only a very small number of the eight possible alternative organizations is actually used, when measured by the point at which Ss start to track the sequences manually. This result has been replicated with slow and fast patterns, using direct verbal descriptions, by Gamer and Gottwald (1967, 1968). With this approach, the nature of the preferred organization is expected to shed light on the psychological processes that enable the organism to deal with repeating sequences.

The third approach is concerned also with the perceived organization, but in its variability more than in the particular organization used or perceived. For theoretical reasons, Garner (1962) had suggested that perceptually good patterns should have few alternatives, because a small set of alternatives is more redundant with respect to the total set than is a large set. This relation was first shown to hold for visual patterns (Gamer \& Clement,
1963). In addition, however, Royer and Garner (1966) showed that difficulty of pattern perception (measured by delay before a tracking response) was highly correlated with the variability in number of alternative patterns used by Ss; once again, this relation between difficulty and variability of pattern organization has been shown to hold with slow as well as with fast patterns by Garner and Gottwald $(1967,1968)$.

One result from all research on temporai auditory patterns, and which is thoroughly confirmed in the present research, is that the psychologically meaningful unit for Ss in such experiments is the run of identical elements, not the individual elements themselves. Rarely is a pattern perceived as organized with a break in a run of identical elements, so that the number of possible alternative organizations is more properly specified by the number of runs of identical elements than by the number of elements themselves.

\section{Purpose}

The intent of the present paper is to explore further the principles underlying the preferred organizations of temporal auditory patterns. The most extensive work on this problem was done by Royer and Garner (1966), but their use of patterns of eight elements put restrictions on the kinds of alternative organization that could occur to the extent that the principles established had to be considered somewhat tentative. For example, their data showed that patterns were frequently organized to start at the beginning of a series of single alternations, but with eight-element patterns, a series of at least two single alternations necessarily is also at the end of the longest run in the series. Another suggestion from their data was that when a particular organization is used for one pattern, then if another pattern is the temporal reverse of the first, the preferred organization of the second pattern will be the reverse of the preferred organization of the first. But with eight-element patterns, there are only two pairs of basic patterns that are reversals of each other, so that the generality of the conclusion might again be tentative. Yet, such a result would clearly indicate that the organization was occurring in some 
wholistic manner, rather than as a train of elements, and further investigation is needed.

Because the organizing principles suggested in the previous study are tentative, because there is limited information available in this area of perception, and because at least one theoretical approach emerging from probability learning (Restle, 1967) was found by Royer and Garner to be clearly inapplicable to their results, the course of the present research was to avoid selection of sequences for testing hypothesized principles and to investigate, instead, the perceptual organization of all members of a given class-the class of all possible sequences of nine elements-for any principles that could be extracted. The addition of one element to the sequences of eight elements doubles the number of actual possible sequences and provides a much greater range of alternative organizations across which principles can act.

Direct description of the pattern is the basic measure, and these descriptions are highly ordered. The data are evaluated as evidence that the perceived patterns are temporal Gestalten produced according to principles of balance, symmetry, and simplicity. The structural characteristics of the sequences in terms of the number and variability of run lengths are evaluated for their effects on the perceived pattern.

\section{Technique}

Our previous research on this problem had shown that the preferred or accepted organization was influenced to some extent by the actual starting point of the stimulus sequence, so that all possible starting points had to be used to counterbalance for this effect. With the 512 sequences possible with nine elements, such a procedure is prohibitive. So for this study, a technique was developed that allowed a description to be made easily, in a group situation, and without the necessity of presenting every possible starting point of a sequence. Much like speeding up a tape recording of speech to a point where speech is not perceived but only a timbre-like quality, a binary sequence presented at a rate of perhaps 15 elements per second or more assumes a particular quality, a timbre, that is a different experience from that of a sequential pattern at slower rates. This fact means, however, that it is possible to avoid using all possible starting points of the sequential patterns by beginning a sequence at a very high rate, then reducing the rate to the point where $S$ is able to perceive a sequential pattern. Once the sequence is perceived as an organized pattern, it is a simple matter to further reduce the rate until $S$ is able to write his coding of the sequence while it is still being presented.

\section{METHOD}

\section{Subjects}

A total of 64 Ss, comprising two classes in introductory psychology at Cleveland State University, participated. There were 32 students in each class, and all were seated in their usual positions in the classroom.

\section{Stimuli}

Physical characteristics. The auditory stimuli were produced by two saw-tooth oscillators, each tone going to a separate amplifier and speaker. The frequencies of the two stimuli were 176.4 and $180.9 \mathrm{~Hz}$. (These frequencies matched the fundamentals of the sounds used in the study by Royer and Gamer, 1966.) The distinctive difference between the tones was due primarily to differences in the harmonics of the wave-forms. The two speakers were about 8 in. apart, which was not far enough to allow auditory localization to differentiate the two tones. The intensity of the first tone was set by asking the Ss in the back of the room to indicate a comfortable listening level, and the intensity of the second was then adjusted until the Ss reported, by raising their hands, that the second tone was as loud as the first. The actual sequencing of the tones was accomplished with a digital logic system. The duration of each stimulus element was always one-half of the period between successive stimuli. The rate of presentation was variable from 30 elements per second to less than 1 per second.

Sequences. If all sequences that can become equivalent to other sequences by substituting one element for the other or

Table 1 Descriptions of Recurrent Patterns of Nine Elements Having Four Runs

\begin{tabular}{|c|c|c|c|c|c|c|c|}
\hline \multirow[b]{2}{*}{ Group } & \multicolumn{6}{|c|}{ Rank Order of Description } & \multirow[b]{2}{*}{$\mathbf{u}^{3}$} \\
\hline & 1 & & 2 & 3 & & 4 & \\
\hline I & $1116^{1}$ & $(32)^{2}$ & $6111(27)$ & 1161 & (1) & 1611 (1) & 1.20 \\
\hline II & $\begin{array}{l}2115 \\
5112 \\
5121\end{array}$ & $\begin{array}{l}(38) \\
(28) \\
(31)\end{array}$ & $\begin{array}{ll}5211 & (23) \\
1125 & (26) \\
1215 & (23)\end{array}$ & $\begin{array}{l}1521 \\
2511 \\
2151\end{array}$ & $\begin{array}{l}(1) \\
(7) \\
(4)\end{array}$ & $\begin{array}{l}1152(0) \\
1251(0) \\
1512(2)\end{array}$ & $\begin{array}{l}1.06 \\
1.40 \\
1.45\end{array}$ \\
\hline HII & $\begin{array}{l}3114 \\
4113 \\
1314\end{array}$ & $\begin{array}{l}(35) \\
(30) \\
(23)\end{array}$ & $\begin{array}{l}4311(14) \\
1134(19) \\
4131(18)\end{array}$ & $\begin{array}{l}1143 \\
3411 \\
3141\end{array}$ & $\begin{array}{l}(6) \\
(10) \\
(10)\end{array}$ & $\begin{array}{l}1431(2) \\
1341(0) \\
1413(9)\end{array}$ & $\begin{array}{l}1.44 \\
1.46 \\
1.89\end{array}$ \\
\hline IV & $\begin{array}{l}2214 \\
4122 \\
2124\end{array}$ & $\begin{array}{l}(31) \\
(28) \\
(33)\end{array}$ & $\begin{array}{l}4221(13) \\
1224(23) \\
4212(22)\end{array}$ & $\begin{array}{l}2142 \\
2412 \\
2421\end{array}$ & $\begin{array}{r}(13) \\
(7) \\
(1)\end{array}$ & $\begin{array}{l}1422(1) \\
2241(0) \\
1242(0)\end{array}$ & $\begin{array}{l}1.55 \\
1.41 \\
1.08\end{array}$ \\
\hline $\mathbf{V}$ & $\begin{array}{l}3213 \\
3312 \\
3132\end{array}$ & $\begin{array}{l}(28) \\
(32) \\
(29)\end{array}$ & $\begin{array}{l}2133(19) \\
3123(26) \\
2313(25)\end{array}$ & $\begin{array}{l}3321 \\
1233 \\
1323\end{array}$ & $\begin{array}{l}(8) \\
(2) \\
(5)\end{array}$ & $\begin{array}{l}1332(0) \\
2331(0) \\
3231(3)\end{array}$ & $\begin{array}{l}1.43 \\
1.17 \\
1.54\end{array}$ \\
\hline$\underline{\text { VI }}$ & 2223 & (25) & $3222(24)$ & 2232 & (8) & $2322(5)$ & 1.73 \\
\hline
\end{tabular}

1 The pattern description, in terms of the sequence of runs of different lengths.

2 Frequency of use of the particular description.

Uncertainty in bits of the distribution of descriptions. by altering the starting point are considered to form a single basic pattern, then the 512 possible sequences of nine elements reduce to 30 basic sequences. In the experiment, 26 of these were used. The four sequences not used were (a) one having a single element repeated nine times and (b) three of the sequences having just two runs: 6 and 3,7 and 2 , and 8 and 1 . The majority of the remaining patterns are identified in Tables 1 and 2, with the code indicating the lengths of the runs. To illustrate, the actual sequence XXXXXOXOO is indicated in Table 1 as 5112. The sequences are also identified in groups, each group containing all the basic patterns that have the same distributions of run lengths. Thus, Group 3 contains three basic patterns, each having one run of Length 4 , one of Length 3 , and two of Length 1.

Table 1 shows all the basic sequences that have four runs, and for each of these, there are four possible descriptions or preferred organizations. Table 2 shows all the basic sequences that have six runs, and for each of these, there are six possible descriptions, if we do not consider possible descriptions that start in the middle of a run. The sequences used that are not shown in these two tables are the degenerative 212121 sequence, which is really a sequence with just two runs, the 54 sequence, and one sequence with eight runs, 21111111.

\section{Procedure}

The Ss were told that the experiment was one in the perception of auditory sequences, and that the sequences would be nine elements long. A diagram of the answer sheet, which they had already received, was drawn on the blackboard, with the column headings numbered from 
Table 2

Descriptions of Recurrent Pattems of Nine Elements Having Six Runs

\begin{tabular}{|c|c|c|c|c|c|c|c|c|}
\hline \multirow[b]{2}{*}{ Group } & \multicolumn{7}{|c|}{ Rank Order of Description } & \multirow[b]{2}{*}{$\mathbf{U}^{3}$} \\
\hline & 1 & 2 & 3 & & 4 & 5 & 6 & \\
\hline VII & $111114^{1}(37)^{2}$ & $411111(20)$ & 111141 & (2) & $111411(1)$ & $114111(0)$ & $141111(0)$ & 1.22 \\
\hline VIII & $\begin{array}{l}321111(29) \\
111123(28) \\
121113(33) \\
111213(22) \\
311211(27)\end{array}$ & $\begin{array}{l}211113(25) \\
311112(27) \\
312111(23) \\
311121(19) \\
112113(26)\end{array}$ & $\begin{array}{l}111132 \\
231111 \\
111312 \\
131112 \\
121131\end{array}$ & $\begin{array}{l}(3) \\
(5) \\
(3) \\
(6) \\
(5)\end{array}$ & $\begin{array}{l}111321(0) \\
123111(0) \\
211131(2) \\
213111(5) \\
113112(4)\end{array}$ & $\begin{array}{l}113211(0) \\
112311(0) \\
113121(0) \\
112131(1) \\
211311(1)\end{array}$ & $\begin{array}{l}132111(0) \\
111231(0) \\
131211(0) \\
121311(1) \\
131121(0)\end{array}$ & $\begin{array}{l}1.24 \\
1.33 \\
1.39 \\
1.94 \\
1.69\end{array}$ \\
\hline IX & $\begin{array}{l}212112 \\
211212(34) \\
222111\end{array}$ & $\begin{array}{l}221211(14) \\
112122(14) \\
211122(19)\end{array}$ & $\begin{array}{l}121122 \\
2122116 \\
2211126\end{array}$ & $\begin{array}{l}(6) \\
12) \\
16)\end{array}$ & $\begin{array}{l}112212(2) \\
122112(8) \\
111222(5)\end{array}$ & $\begin{array}{l}122121(2) \\
221121(5) \\
112221(1)\end{array}$ & $\begin{array}{l}211221(0) \\
121221(0) \\
122211(0)\end{array}$ & $\begin{array}{l}1.62 \\
2.17 \\
1.94\end{array}$ \\
\hline
\end{tabular}

I The pattem description, in terms of the sequences of runs of different lengths.

2 Frequency of use of the particuler description.

3 Uncertainty in bits of the distribution of descriptions.

1 to 9 . Then $\mathrm{E}$ explained that a sequence would be presented at a rate that was too fast for the sequence of sounds to be determined; gradually the rate of presentation would be decreased until a pattern of two sounds began to be heard. They were to listen, and, when the sequence was slowed to a very low rate, they were to write out the sequence of sounds of the pattern they heard. At that point, the higher tone was turned on, and Ss were instructed that this particular tone was to be coded by writing an $X$ when it occurred; the other tone was turned on, and Ss were instructed to write an $O$ when that particular tone was heard. Each tone was sounded singly so that Ss learned them thoroughly. It was explained that if a sequence of only the high tone were heard, Ss would write a series of Xs across the paper. Earlier instructions regarding this procedure were repeated.

The first sequence, 212121, was presented. It began at an extremely rapid rate that was gradually reduced; the deceleration was decreased as the rate approached $4.0 / \mathrm{sec}$. After the presentation reached the minimum rate allowable by the programmer, the sequence was continued at that rate until $E$ observed that all of the Ss appeared to be finished. A volunteer was asked to indicate how he had reported the sequence; this was written on the board. E then asked if anyone else had heard the sequence in a different way. The alternative was described. $E$ then wrote the third alternative, which no one had written. The question was asked if there was anyone who had been unable to describe the sequence at all. It was emphasized that there were no right or wrong answers, and that Ss should describe the pattern as they heard it.

Then the 54 sequence was presented in the same fashion; on its completion, E asked if anyone had been unable to describe it. Instructions were given that if $S$ was not able to complete the sequence at the time $E$ had turned off the stimulus, $S$ was to raise his hand and the procedure would be repeated. Repetition was necessary only in the early patterns.

Sequence presentation. After these two practice sequences with patterns having just two runs, the remaining 24 sequences were presented. Each group of Ss received all 24 sequences in an order determined by a table of random numbers. In order to control for the possible effects of actual stimulus frequency, however, each of the two complementary forms for each basic pattern was used, one for each group, assigned on a random basis. Thus, if one group received the 4311 pattern as XXXXOOOXO, the other group received it as OOOOXXXOX.

\section{RESULTS AND DISCUSSION}

The primary data obtained from this experiment are displayed in Tables 1 and 2 . In these tables, the possible descriptions (as sequences of runs) for each basic pattern are listed in order of preference, and the actual frequency with which each description was used is indicated in parentheses next to the description. These frequencies combine descriptions from both forms of each pattern (i.e., the pattern and its complement). The resulting most frequent descriptions are hereafter referred to as preferred patterns. Descriptions that were in error ( $5.4 \%$ of all responses) or that started in the middle of a run (1.9\% of all responses) are omitted.

\section{Effect of Tonal Frequency}

Our previous research (Royer \& Garner, 1966) had shown no effect of the particular stimulus used for each element in the sequence. The present results showed a very strong effect, however, in that $66.8 \%$ of the descriptions began with the higher tore. There are two possible reasons for obtaining this effect in the present experiment, while not obtaining it in the previous one.

First, when the sequences are originally played at a quite fast rate, there is a figure-ground phenomenon in which the two tones appear to pull apart briefly and are perceived as two separate patterns of tones, each with its own time pattern (see Miller \& Heise, 1950). The higher-pitched of the two tones is more easily followed, and as the rate is slowed down, $S$ tends to maintain an organization based on this higher-pitched tone.

Second, the two speakers emitting the tones were very close together, so that lateral localization of the tones was not possible. In the previous experiment, lateral localization was a dominant characteristic of the tones and probably made the use of the frequency cue a very secondary one. Thus, the greater use of the higher-pitched tone in the present experiment is possibly due to the fact that pitch was the only differentiating cue available, and the bias would not have occurred if another differentiating cue, such as laterality, had been available.

This bias produces no serious distortion of the data since it enters in only when the inherent structural properties of the patterns lead to essentially equally good alternatives, i.e., when alternative factors determining organization are weak or not strongly differentiating. General inspection of Tables 1 and 2 will show that for almost every pattern, there are two strongly preferred organizations, and that one of each of these organizations starts with each of the two tonal elements. To illustrate, Pattern 2115 (the preferred organization) is $X \times 0 \times 00000$, but the second preference is 5211 , or O0O00XXOX: thus, the two preferred organizations provide a starting point for each tonal element. The bias to start on the higher note makes the first preference actually much more frequently used than the second $(\mathrm{X}$ being the higher-pitched element). However, half the Ss received the complement of this pattern, and for the complement, the first and second preferred organizations are OOXOXXXXX and

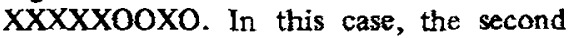
preference was actually used more frequently, since it starts with the higher-pitched tone. This fact, that there are two preferred organizations, one starting at each tonal element, plus the fact that each pattern was used half the time as itself and half the time as its complement, means that a counterbalancing has been achieved so that the bias is offset by the use of the pattern complements. As an example, the degenerative pattern XXOXXOXXO (212121) has three possible descriptions. The frequencies for the elements $X X O$, when $X$ was the higher tone, were 18,0 , and 14 , respectively; when $O$ was the higher tone, the frequencies were 13,0 , and 19 . The 
important point is that the bias did not induce $S s$ to use organizations not otherwise preferred. It did induce them to choose between alternatives already acceptable, but it was not strong enough to alter the general distrubution of alternatives.

\section{Organizing Principles}

Temporal Gestalt. As mentioned, at extremely rapid rates, sequences of stimuli such as those used here have a unitary perceived pattern, a timbre, which becomes an organized pattern of separate events at lower rates and an unorganized sequence of discrete events at still lower rates. It is in the last case, where only order and not time intervals appear to matter much, that theories have originated emphasizing the unidirectional succession of events in terms of transitional probabilities, Markov chains, and first-, second-, and higher-order rules of runs-structure. A question of general concern in this study, where time intervals do seem to matter, is whether the organizing principles are based on a single-dimensional time sequence in which subsequent events or runs are somehow related only to preceding ones, as might be suggested by the probability-learning work, or are based on the pattern as a totality, with all parts interacting in a forward and backward fashion. The latter question is not irreconcilable with a runs-sequence analysis. The perception may be wholistic, but the stimulus structure may be examined analytically. Runs appear to be the psychologically meaningful units for Ss. As such, a run represents the duration of the temporal occupancy of similar elements. The sequence of runs represents the ordering of alternative events in varying temporal relations. It is possible that these temporal relations are apprehended not by a unidirectional linear progression of elements but by a bidirectional comparison of the various intervals. The concept of rhythm in auditory phenomena implies the evaluation of relative temporal intervals and can be appreciated only in terms of bidirectional analysis; a rhythm is established not only by the present temporal event taken in comparison to the past ones but also by the anticipated events that confirm a possible organization or indicate a new one.

While it is possible that patterns are learned at slow rates in a different way than they are perceived at faster rates (Garner \& Gottwald, 1968, show that this is so to some extent), it is eminently clear from the present data that these temporal patterns are perceived in a wholistic way, with all parts contributing to the nature and acceptability of the particular organization.
The primary evide. : for this statement comes from considera $:$ of the role of pattern reversals ${ }^{3}$ in de nining pattern organization. For every pus. le sequence of nine dichotomous elem, $?$, there is another sequence that is the i rsal of it in time. Thus, sequence XXXOX, 00 has a reversal OOXXXOXXX, and sence XXX00X000 has a rer.ral OOOXOOXXX. This reversal sequic. occurs either within the same basic patt ${ }^{r}$ or within another basic pattern. In the former illustration, it is in the same basic pattern, being the first pattern displaced seven elements to the right; in the latter illustration, it occurs in another basic pattern. In either case, for each possible pattern organization, there is another organization that is the exact temporal reverse of it.

In Tables 1 and 2, all the pairs of basic patterns that provide reversals of each other are bracketed together, there being seven such pairs of patterns altogether. In five of these pairs, the first two pattern organizations in order of preference are exactly the same within the pair except for the reversal, and in the other two (3213 and $3312 ; 121113$ and 111213 ), the first two choices of the patterns are reversals, but the order of choice is different from one pattern to the other. To illustrate the more frequent result, the first two preferred organizations for the first pattern in Group 4 are 2214 and 4221; for the second pattern, which contains the reversals of these, the preferred organizations are 4122 and 1224 , the exact reversals having the same order of preference. This paralleling of preferred organizations even extends into the third and fourth choices, which again usually match up with the reversed pattern and with the same order of preference.

The patterns in Tables 1 and 2 that are not bracketed are those in which the reversal of a pattern is contained within the same basic pattern, and there are nine such patterns. In eight of these nine basic patterns, the first two preferred pattern organizations are reversals of each other. To illustrate, the first preferred organization in Group 1 is 6111 , and the second is 1116; the first preferred organization in Group 5 is 3132, and the second is 2313; and the first preferred organization in Group 8 is 311211 , the second being 112113 . The only exception to this result is in Group 9, where the first two preferred organizations are not reversals of each other.

The data for the two patterns having just two runs, 54 and 21 , necessarily show this result, since the only two possible organizations are reversals of each other. With the one pattern having eight runs, the first two preferred organizations are again reversals of each other, being 21111111 and 11111112 . Thus, these additional cases confirm the general result.

The importance of these results lies in the fact that two patterns that are reversals are similar to each other only if it is assumed that the pattern is perceived as a totality, so that such relations as symmetry can exist. If it is assumed, as most models concerned with pattern learning do assume, that ease of learning depends on the - 'ation of the succeeding element to the 6 : or more elements that have last occ. . . $d$, then choices of patterns that are simila. . each other in a directional sense should $C$ 'hosen. This point is easy to see with spe. ?: examples. If directional similarity is . - important factor, we would expect that $\mathrm{cl}$ " oing one element should not greatiy aft.: the organization. In Group 2, a pre1.:ed organization is OOXOXXXXX $(2: 15)$. Directional similarity can be preserved by shifting one element in the patiern to generate the pattern OXOOXXXXX (1125). We find, however, that this directionally similar organization of the latter pattern is not the first but the second preference. Instead, the preferred organization is XXXXXOXOO (5112), and this is the exact temporal reverse of the first mentioned sequence, 2115 .

As another example, in Group 9, XXOXXOXOO (212112) is a preferred organization, and in the set of reversals a similar pattern exists: XXOXXOOXO (212211), this pattern being the same as the former with the interchange of an XO toward the end of the pattern. This second pattern, which exists in the set of reversal patterns, and which is similar in the temporal succession of runs, is not the preferred pattern; rather, it is the complete reversal of the former patterns, OOXOXXOXX (211212), which is the preferred organization. While directional similarity is preserved by these operations, the relative temporal occupancy of similar elements is not. Reversals, on the other hand, do not alter the relative temporal occupancy. Thus, if we expect similar patterns to be chosen as preferred organizations, then we have to allow similarity to be defined in terms of total patterns.

Another line of evidence that leads to the conclusion that wholistic organization is important lies in comparisons of organizations of patterns of eight and nine elements. We can consider a particular pattern of eight elements, see what its preferred organization is from Royer and Garner (1966), and then consider each of the possible patterns of nine elements that can be generated by adding one element to 
each of the runs in the pattern of eight elements. There are far too many such comparisons for us to make here, but a single illustration will demonstrate that pattern organization is very little affected by changes in any single run. As the illustration, the preferred organization is 4112 for one of the patterns of eight elements. If we add one element to each of the runs, we obtain preferred organizations of 5112, 4122, and 4113 for three cases, showing no change in the preferred organization. In one case, the preferred organization changes to 2124 , and this occurs when the element is added to the run that was the second preferred starting run in the pattern of eight elements. Thus, changing of a single run in the sequence has little effect on the nature of the preferred organization (or relative temporal occupancy), a fact that once again argues for a wholistic interpretation of the organizing principles.

Balance and directional simplicity. The argument just made is that the basic organizing principles are wholistic in nature. The particular principles that seem most to be operating with these patterns are principles that are wholistic in nature, in that balance over the entire sequence and an ordinal simplicity regarding length of runs seem to be the major organizing principles, and neither of these principles can be stated except in terms of the entire pattern.

Clearly, there is some form of choice of organizing principle, and the primary principle is that the pattern should be organized so that it is balanced (i.e., symmetrical) over its entire length, but with long runs being at the ends. This principle operates wherever it is feasible, and nearly always produces the preferred organization. For example, 2115 and 5112 , 3114 and 4113,2214 and 4122 , and 3213 (although not 3312) are all organizations in which the two longest runs are at the ends of the patterns. Note that symmetry alone is not sufficient, since patterns such as 1521 and 1251,1431 and 1341 , etc., are equally symmetrical but are very unpreferred organizations. Note also that this principle is more difficult to apply with sequences having six runs, although the second preferences of 211113 and 311112 are examples, and 212112 and 211212 are as symmetric as these patterns can be.

Certainly, symmetry as an organizing principle continues to appear dominantly in all research on pattern perception, yet its inadequacy as a sole principle (even with the more detailed concept of subsymmetry, as suggested by Alexander \& Carey, 1968) is apparent. Thus, the more inclusive concept suggested by Garner (1962) of number of alternatives appears
Table 3

Mean Probability (p) and Rank Order (R) of Description According to Organizing Principles of Balance and Ordinal Progression

\begin{tabular}{|c|c|c|c|c|c|c|c|c|c|}
\hline \multirow{2}{*}{\multicolumn{2}{|c|}{$\begin{array}{l}\text { Balance } \\
\text { Class p } \\
\end{array}$}} & \multirow{3}{*}{$\frac{\begin{array}{c}\text { (End) } \\
\mathrm{R}\end{array}}{1.3}$} & \multirow{3}{*}{$\frac{\begin{array}{c}\text { Balance } \\
\mathrm{p}\end{array}}{.039}$} & \multirow{3}{*}{$\frac{\begin{array}{c}\text { (Middle) } \\
\mathbf{R}\end{array}}{3.7}$} & \multirow{2}{*}{\multicolumn{2}{|c|}{$\begin{array}{c}\text { Ordinal Progression } \\
\mathbf{p}\end{array}$}} & \multicolumn{2}{|c|}{ Other } & \multirow[b]{2}{*}{ Members } \\
\hline & & & & & & & p & $\mathbf{R}$ & \\
\hline $\mathbf{A}$ & .504 & & & & .315 & 2.0 & .141 & 3.0 & $\begin{array}{c}5112,2115 \\
4113,3114 \\
4122,2214 \\
3213,3123 \\
311112,211113\end{array}$ \\
\hline $\mathbf{B}$ & .466 & 1.0 & .034 & 5.5 & & & .500 & 3.6 & 212112,211212 \\
\hline C & & & & & .781 & $*$ & .219 & $*$ & $\begin{array}{l}222111,6111 \\
3222,411111 \\
21111111\end{array}$ \\
\hline
\end{tabular}

* Not calculated (see text)

still to be a useful unifying concept, particularly when we consider that the second organizing principle is one of maximum asymmetry.

The second principle, which seems to be applied as a secondary choice, is that the pattern should increase or decrease in nun length in as regular a fashion as the particular pattern permits. Thus, 5211 and 1125,4311 and 1134,4221 and 1224 are preferred organizations, as are also 321111 and 111123 . The importance of the regular progression in run length can be seen by noting that pattern organizations such as 1143 and 3411 are much less preferred than their alternatives, 4311 and 1134 However, in Group 5, there is an anomaly in that 3321 and 1233 are much less preferred than 2133 and 3312 , which are not very well balanced patterns either.

Detailed examination of the data suggests other secondary organizing principles, but it is questionable that much generality can be established for them with sequences of nine elements only. Note also that the apparent conclusion obtained from the more limited set of patterns obtainable with eight elements, that there is a preference to start patterns with runs of single alternations, does not seem to hold up, since such organizations are either not chosen or are explainable on the basis of increasing length of run. Thus, Pattern 111123 is a preferred pattern but 111132 is not, the difference being in the relation of the 2 and 3 runs, not in the beginning alterations.

\section{Summary of Organizing Principles}

In Table 3, the mean rank order and mean probability of descriptions, according to the organizing principles of balance and directional simplicity, are contrasted with all other possible descriptions. There are 10 patterns (five reversal pairs) that can be described so that there is a balance over their entire length with either long or short runs at the ends, and so that there is a regular ordinal progression of run lengths. These are categorized as Class $\mathbf{A}$. There are 2 patterns (a reversal pair) that can be described with respect to balance but not according to ordinal progression; these are categorized as Class B. There are 6 pattems that can be described according to ordinal progression and not according to balance; these are categorized as Class $C$. Mean rank order was not calculated for Class $\mathrm{C}$ since there are patterns that are reversals internally, e.g., 3222 and 2223 ; hence, two ranks would be averaged for each pattern instead of only one, as in the other cases.)

It is clear that balance or symmetry with organizing principle when available. If it is not available but ordinal progression is, the latter principle will be expected over other alternatives.

There are eight remaining patterns that do not meet the criteria for inclusion in Table 3. However, many of these and others listed above (a total of 18 patterns) can be evaluated according to run length, i.e., whether the descriptions begin with the longest run or immediately after the longest run. The probability of a description beginning with the longest run is .412; the probability of beginning at the element immediately after the longest run is .474 . Thus, $88.6 \%$ of the descriptions will begin at one or another of these alternatives.

In this experiment, we have no direct measure of the difficulty with which these patterns are "learned" or perceived (errors being too few to provide such a measure); thus, we cannot relate these organizing principles directly to pattern difficulty. However, Royer and Garner (1966) and Garner and Gottwald $(1967,1968)$ have shown that variability of pattern organization is related both to difficulty of pattern learning and to difficulty of perceptual organization. Thus, information available from this experiment about variability of organization should provide some evidence concerning pattern difficulty.

In Tables 1 and 2 , the uncertainty in bits of the distribution of pattern discriptions is shown for each basic pattern. While this measure is not ideal, due to its great longer runs at the ends is a major

\section{Variability of Organizations}


Table 4

Variability of Pattern Description

\begin{tabular}{cccc}
$\begin{array}{c}\text { Pattern } \\
\text { Length } \\
\text { (in runs) }\end{array}$ & $\begin{array}{c}\text { Length of } \\
\text { Longest } \\
\text { Run (in } \\
\text { elements) }\end{array}$ & $\begin{array}{c}\text { Number } \\
\text { of } \\
\text { Patterns }\end{array}$ & $\begin{array}{c}\text { Uncertainty } \\
\text { (in bits) }\end{array}$ \\
\hline \multirow{4}{*}{4} & 6 & 1 & 1.20 \\
& 5 & 3 & 1.30 \\
& 4 & 6 & 1.47 \\
& 3 & 4 & 1.47 \\
& & 14 & \multicolumn{2}{c}{1.42} \\
6 & 4 & 1 & 1.22 \\
& 3 & 5 & 1.52 \\
& 2 & 3 & 1.91 \\
8 & 2 & 9 & \multicolumn{2}{c}{1.62} \\
& & 1 & 1.66 \\
& & & \multicolumn{2}{c}{1.66} \\
\hline
\end{tabular}

sensitivity to small frequencies, it is the best summary statistic available to describe the variability of a nonmetric distribution.

There are three factors that affect the variability of the descriptions.

Number of runs. The importance of runs as psychological units is demonstrated in Table 4 . The average description uncertainty increases from 1.42 for patterns with four runs to 1.66 for the pattern having eight runs. But considering how small these differences are, the importance of other factors is demonstrated even more.

Variability of run length. For a given number of runs, the smaller the difference between the shortest and the longest run, the greater the variability of descriptions. This is shown clearly in Table 4 . Thus, the range of length of runs is at least as important a factor as the number of runs.

Availability of organizing principle. The relation between the organizing principles of balance and directional simplicity to variability of description is most easily seen by considering patterns where neither of these principles can operate. To illustrate, in Group 3, balance can operate with 3114 and 4113 , and the secondary principle of directional simplicity can operate with the alternative descriptions of 4311 and 1134 . The third arrangement of these run lengths (e.g., 1314), however, prevents either of these principles from operating, with the result that all four possible pattern organizations of this arrangement are used with rather high frequency. A similar situation holds for Patterns 5121 and 3132.

Description uncertainty for the 10 patterns satisfying both principles (Class A of Table 3 ) is 1.35 ; description uncertainty is 1.57 for the 7 patterns from the same groups having the same run lengths but not satisfying the principles.

In general, we would expect the variability of description to be high if no good organizing principles can be applied or found. However, it is also possible that any organization satisfies the principles, so that high variability will occur also when there are too many good organizations available. To illustrate, 2223 is used with all four of its possible descriptions, but in this case, symmetry or balance is never seriously violated. Thus, all of these organizations should be equally easy rather than equally difficult. Likewise, all three of the patterns in Group 9 produce high variability of description, and again, in these cases, it is difficult not to satisfy the balance and/or directional simplicity principles to a reasonable degree.

Thus, the relation between difficulty and variability of description previously found may be a limited one, with the more general principle being that patterns with few alternative organizations will be easy to learn or to perceive, but those with many may or may not be difficult depending on the reason for the high variability of descriptions.

\section{CONCLUSION}

A concluding remark about the role of organization as being in the stimulus, as opposed to the idea that organization is imposed on the stimulus by the organism, seems in order. The analyses shown here, with complete description of all patterns of a specified class, make it evident that each pattern out of the set has its own set of alternative organizations. The number of runs and the variability of run length were shown to influence the variability of pattern description, which is to say that these properties, which specify stimulus organization, affect the range of alternative organizations that the perceiver will use. Furthermore, the analyses of the $\mathrm{Os}^{\prime}$ descriptions demonstrate that balance and directional simplicity are organizing principles. Neither of these principles can be stated except in terms of the entire pattern. Thus, it is proper to speak of the structure of a stimulus and to ascribe to the stimulus a role in pattern organization.

In the process of perceiving, the organism selects an organization already inherent in the stimulus. The limited organizations possible with a given pattern are made known to the organism, which then selects the one most suited to his preferred organizing principles. With this point of view, and with the results shown here, clearly the organism becomes aware of the entire pattern before selecting a preferred organization. Thus, the concept of his perceiving it as an accumulation of directional associations between sequential elements or, even more so, as associations between the elements and possible responses is quite inadequate. That this conception of the organism perceiving the pattern as a whole and then selecting organizations, or forming hypotheses based on the whole, is not limited to fast perception as has been shown by Garner and Gottwald (1967) in the learning of temporal patterns at slow rates.

The function of the ear is not to impose organization but to discern the structure that does exist in the acoustic environment. Undeniably, there are exceptional circumstances when it is appropriate to say that the $O$ has imposed order, as when a single uniformly repeated sound assumes an organization with every other sound attaining a perceived emphasis. However, the results of this and related studies make it evident that selection of alternative possible organizations is the rule.

\section{REFERENCES}

ALEXANDER, C., \& CAREY, S. Subsymmetries. Perception \& Psychophysics, 1968, 4, 73-77. GALANTER, E. H., \& SMITH, W. A. S. Some experiments on a simple thought-problem. American Journal of Psychology, 1958, 71 , 359-366.

GARNER, W. R. Uncertainty and structure as psychological concepts. New York: Wiley, 1962.

GARNER, W. R., \& CLEMENT, D. E. Goodness of pattern and pattern uncertainty. Joumal of Verbal Learning \& Verbal Behavior, 1963, 2, 446-452.

GARNER, W. R., \& GOTTWALD, R. L. Some perceptual factors in the learning of sequential patterns of binary events. Joumal of Verbal Learning \& Verbal Behavior, 1967, 6, 582-589.

GARNER, W. R., \& GOTTWALD, R. L. The perception and learning of temporal patterns. Quarterly Journal of Experimental Psychology, 1968, 20, 97-109.

MILLER, G. A., \& HEISE, G. A. The trill threshold. Journal of the Acoustical Society of America, 1950, 22, 637-638.

RESTLE, F. Grammatical analysis of the prediction of binary events. Journal of Verbal Learning \& Verbal Behavior, 1967, 6, 17-25.

ROYER, F. L., \& GARNER, W. R, Response uncertainty and perceptual difficulty of auditory temporal patterns. Perception \& Psychophysics, 1966, 1, 41-47.

VITZ, P. C. Information, run structure and binary pattern complexity. Perception \& Psychophysics, 1968, 3, 275-280.

VITZ, P. C., \& TODD, T. C. A model of learning for simple repeating binary patterns. Joumal of Experimental Psychology, 1967, 75, 108-117.

\section{NOTES}

1. This research was supported by Veteran Administration 8200, Part I funds, and by Grant MH 14229 from the National Institute of Mental Health to Yale University. The authors wish to express their appreciation to Dr. Nick Figgtakis for his assistance in providing Ss for this study.

2. Address: VA Hospital, Brecksville, Ohio 44141.

3. The term, pattern reversal, is perhaps more appropriate to visual than to auditory stimuli. In musicology, the term would be retrograde or "crab" pattern. Since the concept of reversal emphasizes the wholistic Gestalt character, it is preferred here.

(Accepted for publication May 15, 1969.) 